OPEN ACCESS

Edited by:

Samer El Hayek,

University of Miami Health System,

United States

Reviewed by:

Kun-Shan Wu,

Tamkang University, Taiwan

Massimo Tusconi,

University of Cagliari, Italy Shabnam Nohesara,

Iran University of Medical

Sciences, Iran

*Correspondence:

Suhaila Ghuloum sghuloum@hamad.qa

Specialty section:

This article was submitted to

Public Mental Health,

a section of the journal

Frontiers in Psychiatry

Received: 09 October 2021 Accepted: 22 November 2021 Published: 13 December 2021

Citation:

Ouanes S, Al-Amin H, Hussein NB, Khan F, Al Shahrani A, David P, Wali AB, Thapur M, Karim MA, Al

Maslamani M, Al-Ansari Z and Ghuloum S (2021) Physical and

Psychosocial Well-Being of Hospitalized and Non-Hospitalized Patients With COVID-19 Compared to the General Population in Qatar

Front. Psychiatry 12:792058. doi: 10.3389/fpsyt.2021.792058

\section{Physical and Psychosocial Well-Being of Hospitalized and Non-Hospitalized Patients With COVID-19 Compared to the General Population in Qatar}

\author{
Sami Ouanes ${ }^{1}$, Hassen Al-Amin ${ }^{2}$, Nurrunnazha Binti Hussein 1, Faisal Khan ', \\ Ahmad Al Shahrani i , Premalatha David ${ }^{1}$, Amel Baker Wali ${ }^{1}$, Maliha Thapur ${ }^{3}$, \\ Mustafa Abdul Karim ${ }^{1}$, Muna Al Maslamani ${ }^{3}$, Zainab Al-Ansari ${ }^{4}$ and Suhaila Ghuloum ${ }^{1 *}$ \\ ${ }^{1}$ Department of Psychiatry, Hamad Medical Corporation, Doha, Qatar, ${ }^{2}$ Weill Cornell Medicine, Psychiatry Department, \\ Doha, Qatar, ${ }^{3}$ Communicable Disease Center, Hamad Medical Corporation, Doha, Qatar, ${ }^{4}$ Weill Cornell Medicine, Medical \\ Education, Doha, Qatar
}

Background: Many studies have shown a high prevalence of depression, anxiety, and stress symptoms in COVID-19 patients and the general population. However, very few studies directly examined the potential impact on the health-related quality of life (HRQoL), and none compared HRQoL in COVID-19 patients to the general population amid the pandemic.

Methods: We carried out a cross-sectional study comparing HRQoL (as measured using the RAND Short Form 36 or SF-36 Health Survey) in randomly selected individuals from three different groups: hospitalized COVID-19 patients, quarantined COVID-19 patients, and controls from the general population in Qatar. We constructed a multivariate analysis of covariance (MANCOVA) to compare the SF-36 scores between the three groups and control for various covariates.

Results: Our sample consisted of 141 COVID-19 inpatients, 99 COVID-19 quarantined patients, and 285 healthy controls. Surprisingly, we found that HRQoL was higher in COVID-19 hospitalized than in COVID-19 non-hospitalized patients than in controls. The main components where COVID-patients scored higher than controls were physical functioning and role limitations due to emotional problems. In COVID-19 patients, the female gender, older age, and past psychiatric history were associated with lower HRQoL.

Conclusions: It seems that COVID-19 patient's HRQoL might be better than expected. Our results can be explained by social support from family and friends, easy access to mental health screening and care, and a possible change of perspectives after recovery from COVID-19, resulting in psychological growth and enhanced resilience.

Keywords: COVID-19, functioning, quality of life, hospitalized, quarantined, general population 


\section{INTRODUCTION}

Amidst the Coronavirus disease (COVID-19) pandemic, more reports showed an increase in the prevalence of depressive, stress, and anxiety symptoms in the general population. Indeed, a metaanalysis of the global prevalence of mental health problems among the general population during the COVID-19 pandemic found a prevalence of $28.0 \%(95 \% \mathrm{CI}=25.0-31.2 \%)$ for depression, $26.9 \%$ (95\% CI $=24.0-30.0 \%$ ) for anxiety, and $36.5 \%$ (95\% CI 30.0-43.3\%) for stress symptoms (1).

A meta-analysis of the prevalence of depression, anxiety, and insomnia symptoms in patients with COVID-19 found a pooled prevalence of $38 \%$ ( $95 \% \mathrm{CI}=25-51 \%)$ for depression, $38 \%(95 \% \mathrm{CI}=24-52 \%)$ for anxiety, and $48 \%(95 \% \mathrm{CI}=$ 11-85\%) for insomnia (2). Similarly, a systematic review of psychiatric sequelae of COVID-19 highlighted high rates of depressive (10.0 and 68.5\%), anxiety (5.0 and 55.2\%), and acute stress reaction (10.0 to $28.0 \%$ ) symptoms, as well as high rates of insomnia (26.0 to $52.2 \%$ ). Even months after recovery, 7.0 to $36.4 \%$ of patients endorsed symptoms suggestive of posttraumatic stress disorder (PTSD), and 40.0 to $69.0 \%$ reported persistent fatigue 2 to 3 months after discharge, with a significant impact on their activities of daily living and quality of life (3). All of these symptoms can affect individual's physical and psychosocial well-being.

However, in stark contrast with the very large number of studies examining depressive, anxiety, and stress symptoms in COVID-19 patients, only a few studies directly examined the health-related quality of life (HRQoL). Some studies reported that HRQoL is low in COVID-19 patients 1-3 months after discharge $(4,5)$; others reported that COVID-19 patients were scoring lower than norms on certain components of the HRQo $(6,7)$.

One fundamental limitation of these studies is the lack of a control arm, and most simply used previously established norms to interpret COVID-19 patient's HRQoL scores (8). Thus, it is better to have a control group to discern the direct effects of COVID-19 infection on HRQoL from the overall impact of the pandemic on the whole population. Indeed, some studies reported high rates of depression, anxiety, and stress in the general population $(1)$, and others found low HRQoL $(9,10)$ in the general population amidst the pandemic.

This study aimed to address some of the shortcomings of the previous studies by (i) directly examining the HRQoL amid the pandemic rather than assuming that depressive, anxiety, and stress symptoms translate into poorer HRQoL; (ii) comparing the HRQoL in COVID-19 patients to a sample from the general population group; (iii) comparing two different groups of COVID-19 patients (hospitalized vs. quarantined).

\section{METHODS}

We conducted this cross-sectional study in July-September 2020 in the State of Qatar to examine HRQoL in COVID-19 patients compared to a sample from the general population. We used hospitalization vs. non-hospitalization as a proxy for COVID19 severity.
We included three groups:

- A group of individuals diagnosed with COVID-19 and admitted to hospital (inpatient group)

- A group of individuals diagnosed with COVID-19 but not requiring hospital admission (quarantine group)

- A general population sample (control group)

We enrolled participants through phone interviews or an anonymous online version of the same questionnaire through Survey Monkey. We used phone interviews for cases tested positive for COVID-19, hospitalized or quarantined. For the general population sample, we sent a link to the online version by phone.

\section{Participants}

The inclusion criteria for the three groups were the following: adults aged 18-65 years who could speak Arabic or English. For the inpatient group, we excluded patients at the intensive care unit at the time of the study.

For COVID-19 groups (both inpatient and quarantine), we used the national records of COVID-19 hospitalized or quarantined patients to select the participants randomly. Then, we contacted every $10^{\text {th }}$ name inviting them to participate until we reached the required number.

For the three groups, we chose not to exclude subjects with past history of psychiatric disorders, because: (i) this would allow us to examine the HRQoL in this subpopulation as well, and to examine the effect of past psychiatric history as a potential predictor for the HRQoL in COVID-19 patients vs. controls (ii) using MANCOVA, we can control for the effect of the presence of past history of psychiatric disorders in the comparison between groups.

We allocated the randomly chosen cases to members of the research team, who then contacted the patients by phone. The interviewers were blinded to whether the patients were hospitalized or quarantined to avoid potential bias.

The research team member explained the purpose of the phone call and invited the patient to participate in the study. After granting the consent, we offered participants to conduct the interview either over the phone or using a web-based survey.

We sent text messages through a mobile service operator to 10,000 subjects, randomly selected English and Arabic speakers to recruit the general public group. The text messages contained a brief explanation of the study and a link to the anonymous online survey.

\section{Sample Size Calculation}

We calculated the sample size using the one-way ANOVA to compare three means (scores of SF-36 for each group) followed by pairwise comparisons with two-sided equality. Thus, we had three groups (i.e., three pairwise comparisons). In addition, we wanted a significant difference of $33 \%$ for each comparison, with a standard deviation of 10 , a power of $80 \%$, and a type one error of $5 \%$ (significance level). Based on these parameters, the calculated sample size was 83 subjects for each group. Further, we elected to recruit at least 100 subjects for each group to account for $20 \%$ of dropouts or incomplete data. 


\section{Measurements}

The survey consisted of questions about basic sociodemographic information and sleep and an assessment of the HRQoL using the RAND Short Form 36 (SF-36) Health Survey in English or Arabic (depending on the participant's preferred language).

The RAND SF-36 questionnaire is a widely used 36-item questionnaire to evaluate HRQoL. It covers eight domains of physical and mental well-being: physical functioning, role limitations due to physical health, role limitations due to emotional problems, energy/fatigue, emotional well-being, social functioning, pain, and general health (11).

We interpreted and analyzed the SF-36 scale results using the scoring rules detailed on the RAND corporation Website (RAND Corporation, 12). For each scale, the score ranges from 0 to 100, with 100 indicating the best health. These eight concepts have also been summarized into two scales: a physical component score and a mental component score (13). The questionnaire was constructed for self-administration and by telephone interview (14). The English version of the SF-36 has demonstrated excellent reliability (Cronbach's alpha for the eight scales ranging from 0.85 to 0.94 ) and discriminant validity (15). The Arabic version was previously validated and showed good reliability (median Cronbach's alphas exceeded 0.70 for every scale except for general health, which had an alpha value of 0.6 ), high correlations with the English version (ranging from 0.73 to 0.92 ), as well as good test-retest reliability (16). Previous studies used the Arabic SF-36 in different Arab countries, including Saudi Arabia (16), Jordan (17), Egypt (18), Tunisia (19), Lebanon (20), and Qatar (21).

\section{Ethical Considerations}

Hamad Medical Corporation Institutional Review Board (MRC05-045) approved this study.

Informed consent was obtained from all participants. All the research team members were bilingual (Arabic and English) and received similar training to standardize the consent process. Participants were offered a consultation with mental health services if needed.

\section{Statistical Analysis}

Data were analyzed using SPSS for Windows, version 26.

\section{Descriptive Statistics}

We determined absolute and relative frequencies for categorical variables and the mean and standard deviation (SD) for the continuous ones.

\section{Analytical Statistics}

To compare categorical variables between groups (inpatient group, quarantine group, and controls), we used Pearson's Chisquare and, in case of non-validity (cells with an expected count $<5$ ), Fischer's exact test. To compare continuous variables between groups (pairwise), we used the $t$-test for independent samples.

We chose to use the APA style in the table reporting chi-square and $t$-test results because the high number of comparisons would otherwise make the table too complex, and difficult to read.
We used a one-way analysis of variance to examine the associations between the SF-36 physical and mental component scores and the categorical variables. For the variables that did not follow a normal distribution (as per the Shapiro-Wilk test), we used non-parametric correlations to examine the associations between the SF-36 physical and mental component scores and the continuous sociodemographic and clinical variables.

To compare the SF-36 scale scores between the three groups (inpatient, quarantine, and controls), we constructed a multivariate analysis of covariance (MANCOVA): the SF36 scores (physical functioning, role limitations due to physical health, role limitations due to emotional problems, energy/fatigue, emotional well-being, social functioning, pain, general health) as dependent variables; and the group (inpatient vs. quarantine vs. controls) as a fixed factor, with gender, age, nationality group (dichotomized as belonging to the most represented group among COVID-19 patients, i.e., Indian Subcontinent vs. others), education (dichotomized as higher education vs. others), occupation (dichotomized as belonging to the most represented group among COVID-19 patients, i.e., craft and manual workers vs. others), and previous psychiatric history as covariables. We chose to conduct a MANOVA rather than a multiple regression analysis because the SF36 has eight components that cannot be summarized into one total score (12). Preliminary assumptions for MANCOVA (including normality, linearity, univariate and multivariate outliers, covariance matrices, and multicollinearity) were tested. Pillai's trace test was used because the SF-36 scores violated the normality assumption. The effect size was assessed using the partial eta squared. The alpha value was set at 0.05 . Finally, we adjusted $p$ values for multiple comparisons using Bonferroni's method.

\section{RESULTS}

\section{Sociodemographic and Clinical Characteristics}

Our sample consisted of 141 inpatients, 99 quarantined individuals with COVID-19, and 285 controls (subjects from the general population who were neither infected nor quarantined) (Table 1).

The proportion of males was significantly higher in inpatients than in the quarantine sample than in controls. Mean age was also higher in inpatients than in quarantined individuals or controls. More craft and manual workers in the inpatient group than in quarantine than in controls. The inpatient group also had lower education levels than both other groups.

The percentage of participants who reported positive psychiatric history (anxiety, depression, or both) was comparable between groups (ranging between 3 and 4.6\%). The number of sleep h was comparable across groups, with a mean of 7.1-7.2 h, and an SD of 1.3-1.4.

When comparing SF-36 scale scores, we found no significant differences between the inpatient and quarantine groups. However, controls reported lower physical functioning scores than inpatients and quarantined individuals. Similarly, controls 
TABLE 1 | Sociodemographic and clinical features of COVID-19 inpatients vs. COVID-19 infected quarantined individuals vs. controls.

\begin{tabular}{|c|c|c|c|c|}
\hline & & A: Inpatient $n=141$ & B: Quarantine $n=99$ & C: Controls $n=285$ \\
\hline Gender, Male, $n$ (\%) & & $121(85.8 \%)_{a}$ & $69(69.7 \%)_{b}$ & $122(42.8 \%)_{c}$ \\
\hline Age, in years, $m \pm S D$ & & $44.0 \pm 10.5_{a}$ & $36.8 \pm 10.4 b$ & $38.6 \pm 10.2_{b}$ \\
\hline & Arab countries other than Qatar & $16(11.3 \%)_{a}$ & $32(32.3 \%)_{b}$ & $37(13.0 \%)_{a}$ \\
\hline & Indian Subcontinent & $90(63.8 \%)_{a}$ & $58(58.6 \%)_{a}$ & $6(2.1 \%) b$ \\
\hline \multirow[t]{5}{*}{ Occupation, $n(\%)$} & Unemployed & $1(0.7 \%)_{a}$ & $3(3.0 \%)_{a, b}$ & $17(6.0 \%)_{b}$ \\
\hline & Housewife & $6(4.3 \%)_{a}$ & $12(12.1 \%)_{a}$ & $32(11.2 \%)_{a}$ \\
\hline & Craft and Manual Worker & $83(58.9 \%)_{a}$ & $26(26.3 \%)_{b}$ & $8(2.8 \%)_{c}$ \\
\hline & Professional & $45(31.9 \%)_{a}$ & $53(53.5 \%)_{b}$ & $210(73.7 \%)_{c}$ \\
\hline & Student & $3(2.1 \%)_{a}$ & $5(5.1 \%)_{a}$ & $17(6.0 \%)_{a}$ \\
\hline Education, $n(\%)$ & Higher education & $38(27.0 \%)_{a}$ & $54(54.5 \%)_{b}$ & $235(82.5 \%)_{c}$ \\
\hline Past psychiatric history, yes, $n$ (\%) & & $5(3.5 \%)_{a}$ & $3(3.0 \%)_{a}$ & $13(4.6 \%)_{a}$ \\
\hline Number of hours of sleep, $m \pm S D$ & & $7.1 \pm 1.4_{a}$ & $7.2 \pm 1.4_{a}$ & $7.1 \pm 1.3_{a}$ \\
\hline \multirow[t]{8}{*}{ SF-36 scale scores, $\mathrm{m} \pm \mathrm{SD}$} & Physical functioning & $91.0 \pm 15.1_{\mathrm{a}}$ & $91.2 \pm 14.7 \mathrm{a}$ & $79.6 \pm 25.3_{b}$ \\
\hline & Role limitations due to physical health & $79.4 \pm 35.9_{a}$ & $76.3 \pm 38.4 \mathrm{a}$ & $78.4 \pm 32.3_{a}$ \\
\hline & Role limitations due to emotional problems & $83.9 \pm 34.9 a$ & $80.1 \pm 36.2 \mathrm{a}$ & $68.0 \pm 39.5 b$ \\
\hline & Energy/fatigue & $64.3 \pm 21.8_{a}$ & $58.6 \pm 22.7 \mathrm{a}$ & $60.7 \pm 20.5 a$ \\
\hline & Emotional well-being & $77.7 \pm 16.8_{a}$ & $75.2 \pm 18.9_{a, b}$ & $71.0 \pm 20.9_{b}$ \\
\hline & Social functioning & $74.5 \pm 26.6_{\mathrm{a}}$ & $72.3 \pm 28.9_{a, b}$ & $67.6 \pm 26.8_{b}$ \\
\hline & Pain & $83.9 \pm 22.3_{a}$ & $83.1 \pm 23.5_{a}$ & $83.2 \pm 20.0_{a}$ \\
\hline & General health & $77.6 \pm 18.7_{a}$ & $78.9 \pm 17.0_{a}$ & $74.9 \pm 17.0_{a}$ \\
\hline
\end{tabular}

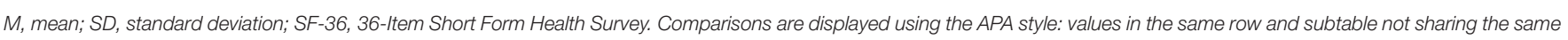
subscript (corresponding to each group in the first row) are significantly different at $p<0.05$. Bonferroni correction was applied to multiple comparisons.

scored worse on role limitations due to emotional problems than the inpatients or the quarantined groups. In emotional well-being and social functioning, controls scored lower than inpatients, but their scores did not significantly differ from quarantined subjects. In other subdomains (role limitations due to physical health, energy/fatigue, pain, and general health), controls did not differ significantly from either of the other groups.

The SF-36 physical component score did not differ between the three groups. Nevertheless, the mental component score was significantly lower in the control group than in the inpatient group. On the other hand, the mental component score in the quarantine group did not differ significantly from either of the other groups.

\section{Factors Associated With SF-36 Component Scores in Patients With COVID-19 (Either Inpatient or Quarantined)}

The SF-36 physical component score was significantly lower in females, and was significantly associated with the nationality group and the occupation, but did not with education level or with past psychiatric history. We also did not find any correlation between the physical component score and age or the reported number of sleep hours (Table 2).

The SF-36 mental component score was significantly lower in participants with past psychiatric history and was positively correlated with the reported number of sleep hours ( $\mathrm{Rho}=0.138$, $p=0.033)$. However, we did not find any association between the SF-36 mental component score and gender, age, nationality group, or education level.

Further, the physical and the mental component scores were not significantly correlated (Rho $=0.062, p=0.342$ ).

\section{Multivariate Analysis}

We used the MANCOVA analysis to compare the SF-36 scores in the three groups controlling for gender, age, past psychiatric history, education, nationality, and occupation. The results (Table 3) showed significant small effects of gender, age, and group (inpatient, quarantine, or controls. Past psychiatric history displayed significant effects on SF-36 scores, whereas education, nationality, and occupation did not show any significant effects. 
TABLE 2 | Factors associated with SF-36 component scores in patients with COVID-19 (either inpatient or quarantined).

\begin{tabular}{|c|c|c|c|c|c|c|c|}
\hline & & Mental component score & $\mathbf{F}$ & $p$-value & Physical component score & $\mathbf{F}$ & $p$-value \\
\hline \multirow[t]{2}{*}{ Gender } & Female & $48.6 \pm 9.2$ & 3.128 & 0.078 & $55.6 \pm 9.1$ & 10.263 & 0.002 \\
\hline & Male & $51.0 \pm 8.2$ & & & $58.8 \pm 5.4$ & & \\
\hline \multirow[t]{5}{*}{ Nationality Group } & Qatar & $48.8 \pm 11.8$ & 0.557 & 0.694 & $53.5 \pm 10.1$ & 3.914 & 0.004 \\
\hline & Arab countries other than Qatar & $49.9 \pm 8.0$ & & & $58.6 \pm 5.1$ & & \\
\hline & Indian subcontinent & $50.7 \pm 8.3$ & & & $58.7 \pm 6.1$ & & \\
\hline & Southeast Asia & $52.4 \pm 5.8$ & & & $57.6 \pm 4.8$ & & \\
\hline & Other & $52.3 \pm 7.4$ & & & $60.6 \pm 3.3$ & & \\
\hline \multirow[t]{5}{*}{ Occupation } & Unemployed & $50.7 \pm 8.8$ & 1.386 & 0.230 & $56.8 \pm 7.1$ & 4.725 & 0.000 \\
\hline & Housewife & $48.8 \pm 10.1$ & & & $59.2 \pm 6.9$ & & \\
\hline & Craft and Manual worker & $51.1 \pm 7.9$ & & & $58.4 \pm 6.6$ & & \\
\hline & Professional & $50.3 \pm 8.2$ & & & $58.0 \pm 5.9$ & & \\
\hline & Student & $53.3 \pm 9.8$ & & & $58.6 \pm 6.2$ & & \\
\hline \multirow[t]{3}{*}{ Education } & Primary or Middle School & $49.8 \pm 9.4$ & 0.415 & 0.661 & $58.0 \pm 7.0$ & 0.090 & 0.914 \\
\hline & Secondary school & $51.1 \pm 7.9$ & & & $58.4 \pm 6.6$ & & \\
\hline & Higher education & $50.5 \pm 8.3$ & & & $58.0 \pm 6.4$ & & \\
\hline \multirow[t]{2}{*}{ Past psychiatric history } & No & $50.8 \pm 8.5$ & 6.479 & 0.012 & $58.3 \pm 6.4$ & 1.617 & 0.205 \\
\hline & Yes & $43.1 \pm 5.2$ & & & $55.3 \pm 5.9$ & & \\
\hline
\end{tabular}

SF-36, 36-Item Short Form Health Survey. SF-36 component scores are shown as mean \pm standard deviation.

TABLE 3 | Multivariate covariance analysis comparing SF-36 scale scores between COVID-19 inpatients vs. COVID-19 infected quarantined individuals vs. controls controlling for sociodemographic and clinical variables.

\begin{tabular}{lcccc}
\hline Effect & Pillai's Trace & $\mathbf{F}$ & $\boldsymbol{p}$-value & Partial Eta squared \\
\hline Gender & $\mathbf{0 . 0 5 3}$ & $\mathbf{3 . 5 6 7}$ & $\mathbf{0 . 0 0 0}$ & $\mathbf{0 . 0 5 3}$ \\
Age & $\mathbf{0 . 0 3 1}$ & $\mathbf{2 . 0 4 5}$ & $\mathbf{0 . 0 4 0}$ & $\mathbf{0 . 0 3 1}$ \\
Past psychiatric history & $\mathbf{0 . 0 7 9}$ & $\mathbf{5 . 4 4 8}$ & $\mathbf{0 . 0 0 0}$ & $\mathbf{0 . 0 7 9}$ \\
Education & 0.021 & 1.355 & 0.214 & 0.021 \\
Nationality & 0.018 & 1.142 & 0.333 & 0.018 \\
Occupation & 0.011 & 0.736 & 0.659 & 0.011 \\
Group & $\mathbf{0 . 0 9 4}$ & $\mathbf{3 . 1 6 0}$ & $\mathbf{0 . 0 0 0}$ & $\mathbf{0 . 0 4 7}$ \\
\hline
\end{tabular}

SF-36, 36-Item Short Form Health Survey. Bold values indicate statistically significant results.

Univariate tests of between-subjects' effects (Table 4) showed that group had a small effect on physical functioning and role limitations due to emotional problems. Age showed a significant small effect on physical functioning, whereas past psychiatric history had small effects on all SF-36 scale scores except physical functioning. Gender showed small effects on the following scale scores: role limitations due to emotional problems, energy/fatigue, emotional well-being, pain, and general health (Figure 1).

\section{DISCUSSION}

In this cross-sectional study, we examined the physical and psychosocial well-being (or HRQoL as measured by the SF-36) of hospitalized and non-hospitalized patients with COVID-19 compared to the general population in Qatar. Surprisingly, we found that HRQoL was higher in COVID-19 hospitalized than in
COVID-19 non-hospitalized patients. The latter also had higher HRQoL than controls. In COVID-19 (both hospitalized and nonhospitalized) patients, the main functioning components that were better than controls consisted of physical functioning and role limitations due to emotional problems. Among COVID19 patients, female participants scored lower in role limitations due to emotional issues, energy/fatigue, pain, and emotional well-being. In COVID-19 patients, older age was associated with lower physical functioning, and past psychiatric history was linked to poorer functioning in all SF-36 domains except physical functioning.

\section{Physical and Psychosocial Well-Being of the General Public Amid the COVID-19 Pandemic}

When compared to the normative data of the Arabic version of SF-36, the general population group had SF-36 scores between one standard deviation below the mean and the mean (17). Most of the previous studies that examined the impact of the pandemic on the general population did not directly assess the HRQoL but rather "assumed" that the HRQoL was affected because they found a high prevalence of depressive, anxiety, and stress symptoms $(22,23)$. For example, one Chilean study that directly assessed the HRQoL in the general population found the HRQoL to be affected in 1,082 adults, between 18 and 60 years old, who were quarantined by the COVID-19 health alert but who were neither confirmed nor suspected cases of COVID-19 (9). Similarly, in a group of Italian non-infected women aged between 28 and 50, SF-36 scores were significantly decreased (10). However, a Dutch study did not find the level of mental well-being during the peak of COVID-19 to be lower than in 2018 (24). This discrepancy could be due to the differences in 
TABLE 4 | Univariate tests of between-subject's effects with SF-36 scale scores as dependent variables, group (inpatients vs. COVID-19 infected quarantined individuals vs. controls) as a fixed factor, and sociodemographic and clinical characteristics as covariables.

\begin{tabular}{|c|c|c|c|c|c|c|}
\hline & Dependent Variable & Type III Sum of Squares & Mean Square & $\mathbf{F}$ & $p$-value & Partial Eta Squared \\
\hline \multirow[t]{8}{*}{ Gender } & Physical functioning & 419.757 & 419.757 & 0.945 & 0.331 & 0.002 \\
\hline & Role limitations due to physical health & 741.223 & 741.223 & 0.634 & 0.426 & 0.001 \\
\hline & Role limitations due to emotional problems & 17599.406 & 17599.406 & 13.018 & 0.000 & 0.025 \\
\hline & Energy/fatigue & 7243.418 & 7243.418 & 17.260 & 0.000 & 0.032 \\
\hline & Emotional well-being & 2715.770 & 2715.770 & 7.646 & 0.006 & 0.015 \\
\hline & Social functioning & 2437.598 & 2437.598 & 3.380 & 0.067 & 0.007 \\
\hline & Pain & 3887.007 & 3887.007 & 8.857 & 0.003 & 0.017 \\
\hline & General health & 2536.761 & 2536.761 & 9.059 & 0.003 & 0.017 \\
\hline \multirow[t]{8}{*}{ Age } & Physical functioning & 3129.109 & 3129.109 & 7.046 & 0.008 & 0.013 \\
\hline & Role limitations due to physical health & 1958.734 & 1958.734 & 1.674 & 0.196 & 0.003 \\
\hline & Role limitations due to emotional problems & 4557.089 & 4557.089 & 3.371 & 0.067 & 0.006 \\
\hline & Energy/fatigue & 782.612 & 782.612 & 1.865 & 0.173 & 0.004 \\
\hline & Emotional well-being & 122.332 & 122.332 & 0.344 & 0.558 & 0.001 \\
\hline & Social functioning & 1.251 & 1.251 & 0.002 & 0.967 & 0.000 \\
\hline & Pain & 258.653 & 258.653 & 0.589 & 0.443 & 0.001 \\
\hline & General health & 14.175 & 14.175 & 0.051 & 0.822 & 0.000 \\
\hline \multirow[t]{8}{*}{ Past psychiatric history } & Physical functioning & 1314.742 & 1314.742 & 2.961 & 0.086 & 0.006 \\
\hline & Role limitations due to physical health & 6587.292 & 6587.292 & 5.630 & 0.018 & 0.011 \\
\hline & Role limitations due to emotional problems & 14181.776 & 14181.776 & 10.490 & 0.001 & 0.020 \\
\hline & Energy/fatigue & 7656.771 & 7656.771 & 18.244 & 0.000 & 0.034 \\
\hline & Emotional well-being & 11106.885 & 11106.885 & 31.272 & 0.000 & 0.057 \\
\hline & Social functioning & 8673.863 & 8673.863 & 12.028 & 0.001 & 0.023 \\
\hline & Pain & 2205.210 & 2205.210 & 5.025 & 0.025 & 0.010 \\
\hline & General health & 7609.925 & 7609.925 & 27.176 & 0.000 & 0.050 \\
\hline \multirow[t]{8}{*}{ Education } & Physical functioning & 1012.351 & 1012.351 & 2.280 & 0.132 & 0.004 \\
\hline & Role limitations due to physical health & 259.209 & 259.209 & 0.222 & 0.638 & 0.000 \\
\hline & Role limitations due to emotional problems & 25.336 & 25.336 & 0.019 & 0.891 & 0.000 \\
\hline & Energy/fatigue & 143.529 & 143.529 & 0.342 & 0.559 & 0.001 \\
\hline & Emotional well-being & 0.024 & 0.024 & 0.000 & 0.993 & 0.000 \\
\hline & Social functioning & 287.095 & 287.095 & 0.398 & 0.528 & 0.001 \\
\hline & Pain & 642.052 & 642.052 & 1.463 & 0.227 & 0.003 \\
\hline & General health & 943.465 & 943.465 & 3.369 & 0.067 & 0.006 \\
\hline \multirow[t]{8}{*}{ Nationality group } & Physical functioning & 43.374 & 43.374 & 0.098 & 0.755 & 0.000 \\
\hline & Role limitations due to physical health & 2621.681 & 2621.681 & 2.241 & 0.135 & 0.004 \\
\hline & Role limitations due to emotional problems & 2098.261 & 2098.261 & 1.552 & 0.213 & 0.003 \\
\hline & Energy/fatigue & 65.496 & 65.496 & 0.156 & 0.693 & 0.000 \\
\hline & Emotional well-being & 163.234 & 163.234 & 0.460 & 0.498 & 0.001 \\
\hline & Social functioning & 303.236 & 303.236 & 0.421 & 0.517 & 0.001 \\
\hline & Pain & 5.768 & 5.768 & 0.013 & 0.909 & 0.000 \\
\hline & General health & 1027.364 & 1027.364 & 3.669 & 0.056 & 0.007 \\
\hline \multirow[t]{8}{*}{ Occupation } & Physical functioning & 354.009 & 354.009 & 0.797 & 0.372 & 0.002 \\
\hline & Role limitations due to physical health & 1189.128 & 1189.128 & 1.016 & 0.314 & 0.002 \\
\hline & Role limitations due to emotional problems & 1434.500 & 1434.500 & 1.061 & 0.303 & 0.002 \\
\hline & Energy/fatigue & 11.276 & 11.276 & 0.027 & 0.870 & 0.000 \\
\hline & Emotional well-being & 100.268 & 100.268 & 0.282 & 0.595 & 0.001 \\
\hline & Social functioning & 1057.067 & 1057.067 & 1.466 & 0.227 & 0.003 \\
\hline & Pain & 209.782 & 209.782 & 0.478 & 0.490 & 0.001 \\
\hline & General health & 104.485 & 104.485 & 0.373 & 0.542 & 0.001 \\
\hline \multirow[t]{8}{*}{ Group } & Physical functioning & 7829.189 & 3914.595 & 8.815 & 0.000 & 0.033 \\
\hline & Role limitations due to physical health & 2339.098 & 1169.549 & 1.000 & 0.369 & 0.004 \\
\hline & Role limitations due to emotional problems & 13550.684 & 6775.342 & 5.012 & 0.007 & 0.019 \\
\hline & Energy/fatigue & 1638.254 & 819.127 & 1.952 & 0.143 & 0.008 \\
\hline & Emotional well-being & 646.024 & 323.012 & 0.909 & 0.403 & 0.004 \\
\hline & Social functioning & 1078.017 & 539.008 & 0.747 & 0.474 & 0.003 \\
\hline & Pain & 1527.752 & 763.876 & 1.741 & 0.176 & 0.007 \\
\hline & General health & 195.708 & 97.854 & 0.349 & 0.705 & 0.001 \\
\hline
\end{tabular}

Bold values indicate statistically significant results. 

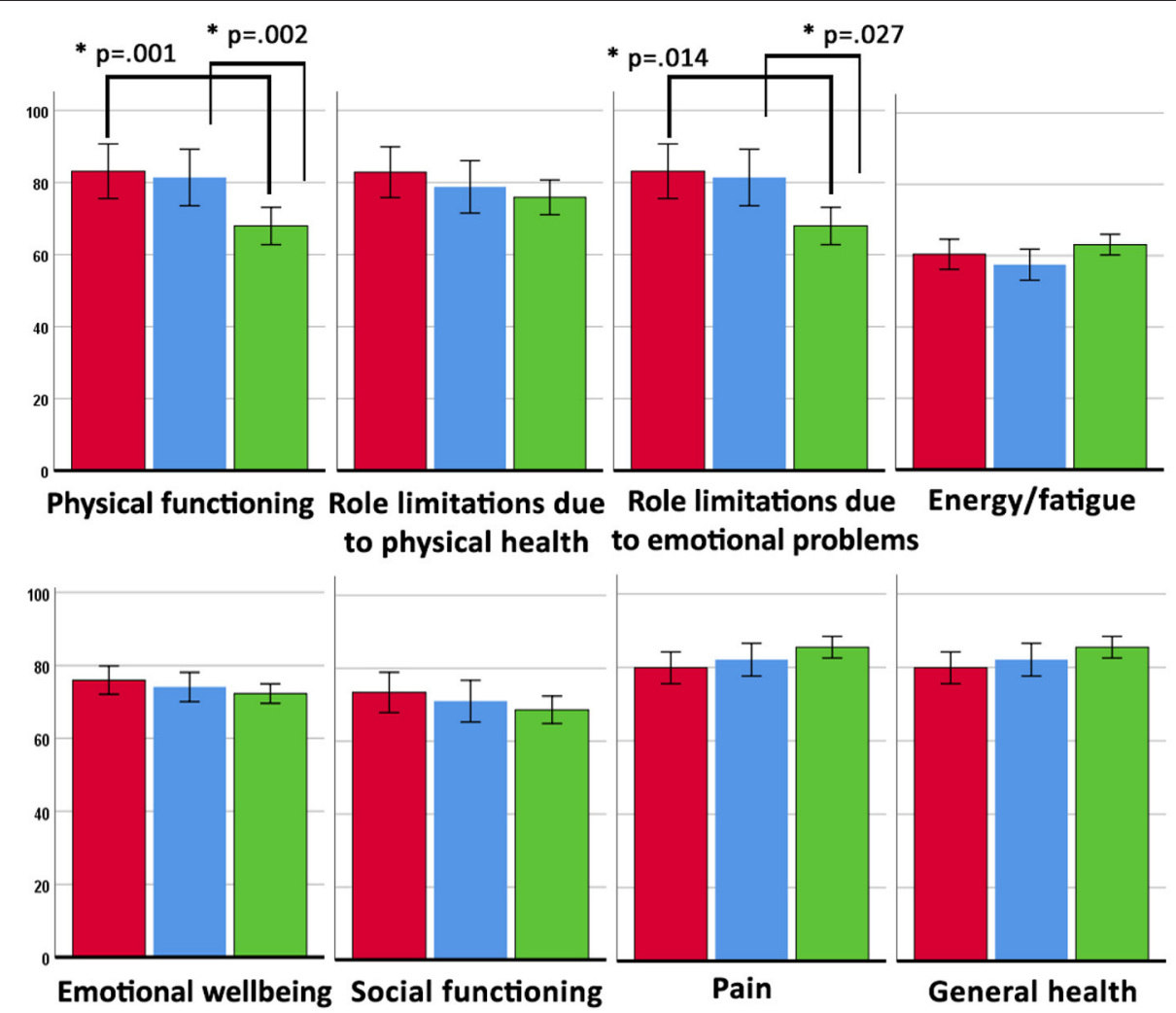

Inpatients

Quarantine

Controls

FIGURE 1 | Estimated marginal means (with their 95\% error bars) of the SF-36 scores in the inpatient, quarantine, and control groups, controlling for gender, age, past psychiatric history, education, nationality, and occupation. All $p$ values were adjusted for multiple comparisons using the Bonferroni method.

participant selection criteria, the times the studies took place regarding the pandemic course, and the variations in COVID19-related restrictions from country to country throughout the pandemic. Overall, there seems to be a paucity of data regarding the potential impact of the pandemic on the general population's HRQoL, even though most of the available indirect evidence suggests a likely negative impact $(1,23)$.

\section{Physical and Psychosocial Well-Being of Patients With COVID-19}

The results of our study showing that COVID-19 patients might not have a poorer HRQoL than controls might be unexpected. Indeed, a growing number of studies showed a high prevalence of depression, anxiety, stress, and insomnia symptoms in patients with COVID-19 $(2,3)$. Based on these findings, it is often "assumed" that COVID-19 infection negatively affects physical and psychosocial well-being. However, most of these studies lacked a control group, and only a few directly examined the impact of the disease on the patient's quality of life and functioning. Thus, one can argue that while the prevalence of depressive, anxiety, and stress symptoms are high in COVID-19 patients, these can also increase in the general population amid the pandemic $(1,25)$. In addition, studies directly comparing infected to non-infected individuals found that infected ones had more pronounced depressive and anxiety symptoms (26), including one study from Qatar (27), but this was not the case for other studies (23).

Moreover, recent studies suggested that the prevalence of depressive and anxiety symptoms in COVID-19 patients is overestimated due to possible overlaps between these symptoms and certain features of the COVID-19 infection, including fatigue, loss of appetite, sleep disturbance, pain, and palpitations (28). In this regard, studies using the Hospital Anxiety and Depression Scale (HADS) (29), designed to identify emotional symptoms of depression in patients with concurrent somatic illness, reported a lower prevalence of depression than those using the Patient Health Questionnaire (PHQ-9), designed to cover all bodily and emotional features (2). Thus, it seems likely that the high prevalence of depression, anxiety, and stress has affected the population as a whole, rather than COVID-19 infected patients in particular.

It is hypothesized that COVID-19 infected patients might have depressive and anxiety symptoms due to the virus's potential neurotropic effects, the immune response, and the isolation due to hospitalization or quarantine $(2,30)$. However, the biological effects of the virus on the brain are not possibly of 
clinical significance in most patients. Previous studies, including a meta-analysis of longitudinal studies and natural quarantine experiments, have not found that quarantine had any major impact on mental health $(31,32)$. It is also possible that infected individuals have benefited from more support from their families, friends, and frequent mental health screening or interventions (23).

In addition, our samples had diverse sociocultural backgrounds, and the distribution of nationalities among the groups was different. Such cultural variation might have impacted the SF-36 scores since the expression of emotions and tendency toward somatization can greatly differ from culture to culture (33).

The mean SF-36 scores for physical and emotional components in our study were comparable to the scores reported in other studies (4-7). Out of these four studies, two interpreted the HRQoL in COVID-19 as being "low." However, the patients' Warwick-Edinburgh Mental Well-being Scales (WEMWBS) did not differ from the population norms (6). Chen et al. (7) findings were even closer to ours: they found that one month after discharge, COVID-19 patients scored lower than the Chinese population norm only in certain HRQoL domains (social functioning and role limitations due to physical and emotional problems). However, they scored higher on other domains (mental health, bodily pain, vitality, general health) with no difference for physical functioning compared to population norms (7).

These findings, including ours, suggest that while the HRQoL in COVID-19 patients is probably affected, it is not necessarily more so than the general population amid the pandemic. In this context, the HRQoL was found to be less affected in COVID19 patients than in their family members (34). Furthermore, the anticipation of the infection might cause more psychological distress than the infection itself since worrying about a negative event is often more anxiogenic than the occurrence of the event itself (35).

Even though most assume that hospitalized COVID-19 patients may experience higher rates of depression, anxiety, and stress, than non-hospitalized patients, other findings did confirm this. Indeed, previous studies reported the prevalence of depressive, anxiety, and PTSD symptoms in never-hospitalized COVID patients to be similar or even higher than in hospitalized patients (De $(3,36-39))$. It is possible that being hospitalized in a protective environment helped to reassure the patients. COVID-19 inpatients reported medical staff care as the main supportive factor as it gave them "a sense of security" (40). Hospitalized COVID-19 patients may also have been more commonly screened for mental health issues and benefited from mental health services during their stay (39). In addition, going through the experience of a potentially severe illness and recovering from it can make people cherish the good aspects of their life, resulting in a positively biased perception of their HRQoL. Such an initial "euphoria" has been reported in patients who survived critical medical conditions (41) and in Ebola survivors (42). In a study examining the psychological experience of COVID-19 patients during a hospital stay, most patients endorsed how the thought that their lives could have suddenly ended made them realize how valuable their life, their families, and their friends are. In a sense, surviving COVID-19 can change perspectives and enhance psychological growth (40).

\section{Factors Associated With Poorer Physical and Psychosocial Well-Being in Patients With COVID-19}

Our results suggested that female COVID-19 patients may have poorer HRQoL than males, particularly in role limitations due to emotional problems, fatigue, pain, and emotional well-being. These findings align with previous studies among COVID-19 patients showing that the prevalence of depression, anxiety, stress, and insomnia symptoms were higher in women than men $(2,3)$. Similarly, Chen et al. reported that the female gender was associated with poorer physical functioning, bodily pain, and role limitations due to emotional problems $(2,3)$. Furthermore, a similar gender difference is reported at the general population level in most studies using the SF-36 in different countries (4345), including Arab countries (19, 46, 47).

We also found that older age in infected patients was associated with lower physical functioning. This association was also reported previously (7). It was attributed to the poor prognosis of COVID-19 in the elderly (48) and the physiological decline of physical functioning with age in the general population $(44,45)$. In our study, we did not find age to be associated with mental health-related HRQoL. Associations between older age and mental health in COVID-19 patients have been inconsistent, with some studies reporting better (49) and others reporting worse outcomes in the elderly (3).

In the present study, past psychiatric history in COVID19 patients was linked to poorer functioning in all SF-36 domains except physical functioning. This link is expected given that COVID-19 patients with prior psychiatric history have been reported to experience higher levels of anxiety, depression, stress, and sleep disturbance than patients with no psychiatric history (3). In addition, patients with mental illness showed an increased risk of contracting COVID-19 and higher hospitalization rates and death compared to individuals with no history of mental illness (50).

\section{Strengths and Limitations}

The present study is one of the few studies to focus on mental health outcomes in patients with COVID-19 infection. Furthermore, contrary to most other studies about mental health consequences of the COVID-19 disease, which merely examined the prevalence of anxiety, depression, and stress symptoms, the present study scrutinized different domains of psychosocial and physical well-being $(3,25)$.

However, some limitations need to be acknowledged. For example, although we used MANCOVA to control the differences in certain sociodemographic characteristics between the three groups, these variations could still bias the proper comparisons. Moreover, mental health issues or the poor perceived HRQoL may have affected the decision to participate in the survey, especially among controls. This bias might have caused controls 
to score poorer than expected. Social-desirability bias might also have influenced certain participant's answers (51). Besides, we could not capture certain variables that may have affected HR-QoL (severity of COVID-19 beyond the mere need for hospitalization, duration of hospitalization, and the exact time elapsed between discharge and filling the questionnaire). In addition, like most previous studies, the cross-sectional design of the present study does not allow to distinguish between shortterm and long-term effects on well-being in COVID-19 patients. A prospective design could have helped disentangle the acute consequences of the infection from any potential long-term sequelae $(2,3)$.

\section{DATA AVAILABILITY STATEMENT}

The raw data supporting the conclusions of this article will be made available upon request from the corresponding author.

\section{ETHICS STATEMENT}

The studies involving human participants were reviewed and approved by IRB of Hamad Medical Corporation. Written informed consent for participation was not required for this

\section{REFERENCES}

1. Nochaiwong S, Ruengorn C, Thavorn K, Hutton B, Awiphan R, Phosuya C, et al. Global prevalence of mental health issues among the general population during the coronavirus disease-2019 pandemic: a systematic review and meta-analysis. Sci Rep. (2021) 11:10173. doi: 10.1038/s41598-021-89700-8

2. Liu C, Pan W, Li L, Li B, Ren Y, Ma X. Prevalence of depression, anxiety, and insomnia symptoms among patients with COVID-19: a meta-analysis of quality effects model. J Psychosom Res. (2021) 147:110516. doi: 10.1016/j.jpsychores.2021.110516

3. Vanderlind WM, Rabinovitz BB, Miao IY, Oberlin LE, Bueno-Castellano C, Fridman C, et al. A systematic review of neuropsychological and psychiatric sequalae of COVID-19: implications for treatment. Curr Opin Psychiatry. (2021) 34:420-33. doi: 10.1097/YCO.0000000000000713

4. Gonzalez J, Benitez ID, Carmona P, Santisteve S, Monge A, MoncusiMoix A, et al. Pulmonary function and radiologic features in survivors of critical COVID-19: a 3-month prospective cohort. Chest. (2021) 160:18798. doi: 10.1016/j.chest.2021.02.062

5. Weerahandi H, Hochman KA, Simon E, Blaum C, Chodosh J, Duan E, et al. Post-discharge health status and symptoms in patients with severe COVID19. J Gen Intern Med. (2021) 36:738-45. doi: 10.1007/s11606-020-06338-4

6. Arnold DT, Hamilton FW, Milne A, Morley AJ, Viner J, Attwood M, et al. Patient outcomes after hospitalisation with COVID-19 and implications for follow-up: results from a prospective UK cohort. Thorax. (2020) 76:399401. doi: 10.1101/2020.08.12.20173526

7. Chen KY, Li T, Gong FH, Zhang JS, Li XK. Predictors of health-related quality of life and influencing factors for COVID-19 patients, a follow-up at one month. Front Psychiatry. (2020) 11:668. doi: 10.3389/fpsyt.2020.00668

8. Nasserie T, Hittle M, Goodman SN. Assessment of the frequency and variety of persistent symptoms among patients with COVID-19: a systematic review. JAMA Netw Open. (2021) 4:e2111417. doi: 10.1001/jamanetworkopen.2021.11417

9. Guzman-Munoz E, Concha-Cisternas Y, Onate-Barahona A, Lira-Cea C, Cigarroa-Cuevas I, Mendez-Rebolledo G, et al. [Factors associated with low quality of life in Chilean adults during the COVID-19 quarantine]. Rev Med Chil. (2020) 148:1759-66. doi: 10.4067/S0034-98872020001201759 study in accordance with the national legislation and the institutional requirements.

\section{AUTHOR CONTRIBUTIONS}

SG, HA-A, and NH formalized the research concept and proposal. ZA-A supported with literature review, administrative aspects of the research, randomized, and distributed subjects. $\mathrm{NH}, \mathrm{FK}, \mathrm{AA}, \mathrm{PD}, \mathrm{ABW}, \mathrm{MT}$, and MK conducted the survey and entered the data. SO did the statistical analysis and wrote the manuscript. SG and HA-A reviewed and edited the manuscript. SG was responsible for the overall project and supervised all steps associated with the study as the lead principal investigator. MAM supported the project by providing data on patients admitted to either hospital or quarantine facilities. All authors contributed to the article and approved the submitted version.

\section{ACKNOWLEDGMENTS}

The research team acknowledges the generous support of Ooredoo and Barqia Companies in the distribution of the survey to the public.

10. Schiavi MC, Spina V, Zullo MA, Colagiovanni V, Luffarelli P, Rago R, et al. Love in the time of COVID-19: sexual function and quality of life analysis during the social distancing measures in a group of italian reproductiveage women. J Sex Med. (2020) 17:1407-13. doi: 10.1016/j.jsxm.2020. 06.006

11. Hays RD, Sherbourne CD, Mazel RM. The RAND 36-item health survey 1.0. Health Econ. (1993) 2:217-27. doi: 10.1002/hec.4730020305

12. Rand Corporation 36-Item Short Form Survey (SF-36) Scoring Instructions. Available online at: https://www.rand.org/health-care/surveys_tools/mos/36item-short-form/scoring.html (accessed March 30, 2021).

13. Ware JE, Kosinski M. SF-36 Physical And Mental Health Summary Scales: A Manual For Users of Version 1. Quality Metric Incorporated (2001).

14. Ware JE, Sherbourne CD. The MOS 36-item short-form health survey (SF36). I conceptual framework and item selection. Med Care. (1992) 30:47383. doi: 10.1097/00005650-199206000-00002

15. Kazis LE, Lee A, Spiro A3rd, Rogers W, Ren XS, Miller DR, et al. Measurement comparisons of the medical outcomes study and veterans SF-36 health survey. Health Care Financ Rev. (2004) 25:43-58.

16. Coons SJ, Alabdulmohsin SA, Draugalis JR, Hays RD. Reliability of an Arabic version of the RAND-36 health survey and its equivalence to the US-english version. Med Care. (1998) 36:428-32. doi: 10.1097/00005650-199803000-00018

17. Khader S, Hourani MM, Al-Akour N. Normative data and psychometric properties of short form 36 health survey (SF-36, version 1.0) in the population of north Jordan. East Mediterr Health J. (2011) 17:36874. doi: 10.26719/2011.17.5.368

18. Mohammed SA, Shebl AM. Quality of life among egyptian patients with upper and lower limb amputation: sex differences. Adv Med. (2014) 2014:674323. doi: $10.1155 / 2014 / 674323$

19. Salem S, Malouche D, Ben Romdhane H. Tunisian population quality of life: a general analysis using SF-36. East Mediterr Health J. (2019) 25:61321. doi: $10.26719 / \mathrm{emhj} .18 .030$

20. El Osta N, Kanso F, Saad R, Khabbaz LR, Fakhouri J, El Osta L. Validation of the Arabic version of the SF-36, generic questionnaire of quality of life related to health among the elderly in Lebanon. East Mediterr Health J. (2019) 25:706-14. doi: 10.26719/emhj.19.041 
21. Gerber LM, Chiu YL, Verjee M, Ghomrawi H. Health-related quality of life in midlife women in Qatar: relation to arthritis and symptoms of joint pain. Menopause. (2016) 23:324-9. doi: 10.1097/GME.0000000000000532

22. Lau JT, Yang X, Tsui HY, Pang E, Wing YK. Positive mental health-related impacts of the SARS epidemic on the general public in Hong Kong and their associations with other negative impacts. I Infect. (2006) 53:11424. doi: 10.1016/j.jinf.2005.10.019

23. Zhang J, Lu H, Zeng H, Zhang S, Du Q, Jiang T, et al. The differential psychological distress of populations affected by the COVID-19 pandemic. Brain Behav Immun. (2020) 87:49-50. doi: 10.1016/j.bbi.2020.04.031

24. Koppert TY, Jacobs JWG, Geenen R. The psychological impact of the COVID-19 pandemic on Dutch people with and without an inflammatory rheumatic disease. Rheumatology. (2020) 60:3709-15. doi: 10.1093/rheumatology/keaa842

25. Vindegaard N, Benros ME. COVID-19 pandemic and mental health consequences: Systematic review of the current evidence. Brain Behav Immun. (2020) 89:531-42. doi: 10.1016/j.bbi.2020.05.048

26. Wang Y, Kala MP, Jafar TH. Factors associated with psychological distress during the coronavirus disease 2019 (COVID-19) pandemic on the predominantly general population: a systematic review and meta-analysis. PLoS ONE. (2020) 15:e0244630. doi: 10.1371/journal.pone.0244630

27. Reagu S, Wadoo O, Latoo J, Nelson D, Ouanes S, Masoodi N, et al. Psychological impact of the COVID-19 pandemic within institutional quarantine and isolation centres and its sociodemographic correlates in Qatar: a cross-sectional study. BMJ Open. (2021) 11:e045794. doi: 10.1136/bmjopen-2020-045794

28. Grant MC, Geoghegan L, Arbyn M, Mohammed Z, Mcguinness L, Clarke EL, et al. The prevalence of symptoms in 24,410 adults infected by the novel coronavirus (SARS-CoV-2; COVID-19): a systematic review and meta-analysis of 148 studies from 9 countries. PLoS ONE. (2020) 15:e0234765. doi: 10.1371/journal.pone.0234765

29. Hansson M, Chotai J, Nordstom A, Bodlund O. Comparison of two selfrating scales to detect depression: HADS and PHQ-9. Br J Gen Pract. (2009) 59:e283-288. doi: 10.3399/bjgp09X454070

30. Troyer EA, Kohn JN, Hong S. Are we facing a crashing wave of neuropsychiatric sequelae of COVID-19? neuropsychiatric symptoms and potential immunologic mechanisms. Brain Behav Immun. (2020) 87:349. doi: 10.1016/j.bbi.2020.04.027

31. Zhu S, Wu Y, Zhu CY, Hong WC, Yu ZX, Chen ZK, et al. The immediate mental health impacts of the COVID-19 pandemic among people with or without quarantine managements. Brain Behav Immun. (2020) 87:568. doi: 10.1016/j.bbi.2020.04.045

32. Prati G, Mancini AD. The psychological impact of COVID-19 pandemic lockdowns: a review and meta-analysis of longitudinal studies and natural experiments. Psychol Med. (2021) 51:20111. doi: 10.1017/S0033291721000015

33. Zhou P, Critchley H, Garfinkel S, Gao Y. The conceptualization of emotions across cultures: a model based on interoceptive neuroscience. Neurosci Biobehav Rev. (2021) 125:314-27. doi: 10.1016/j.neubiorev.2021.02.023

34. Shah R, Ali FM, Nixon SJ, Ingram JR, Salek SM, Finlay AY. Measuring the impact of COVID-19 on the quality of life of the survivors, partners and family members: a cross-sectional international online survey. BMJ Open. (2021) 11:e047680. doi: 10.1136/bmjopen-2020-047680

35. Grupe DW, Nitschke JB. Uncertainty and anticipation in anxiety: an integrated neurobiological and psychological perspective. Nat Rev Neurosci. (2013) 14:488-501. doi: 10.1038/nrn3524

36. Lorenzo RD, Conte C, Lanzani C, Benedetti F, Roveri L, et al. Residual clinical damage after COVID-19: a retrospective and prospective observational cohort study. PLoS ONE. (2020) 15:e0239570. doi: 10.1371/journal.pone. 0239570

37. Mazza MG, Lorenzo RD, Conte C, Poletti S, Vai B, et al. Anxiety and depression in COVID-19 survivors: role of inflammatory and clinical predictors. Brain Behav Immun. (2020) 89:594600. doi: 10.1016/j.bbi.2020.07.037

38. Van Den Borst B, Peters JB, Brink M, Schoon Y, Bleeker-Rovers CP, Schers $\mathrm{H}$, et al. Comprehensive health assessment three months after recovery from acute COVID-19. Clin Infect Dis. (2020) 73:e1089. doi: 10.1093/cid/ciaa1750
39. Deng J, Zhou F, Hou W, Silver Z, Wong CY, Chang O, et al. The prevalence of depression, anxiety, and sleep disturbances in COVID-19 patients: a metaanalysis. Ann N Y Acad Sci. (2021) 1486:90-111. doi: 10.1111/nyas.14506

40. Sun N, Wei L, Wang H, Wang X, Gao M, Hu X, et al. Qualitative study of the psychological experience of COVID-19 patients during hospitalization. $J$ Affect Disord. (2021) 278:15-22. doi: 10.1016/j.jad.2020.08.040

41. Apitzsch S, Larsson L, Larsson AK, Linder A. The physical and mental impact of surviving sepsis - a qualitative study of experiences and perceptions among a Swedish sample. Arch Public Health. (2021) 79:66. doi: 10.1186/s13690-021-00585-5

42. Karafillakis E, Jalloh MF, Nuriddin A, Larson HJ, Whitworth J, Lees S, et al. 'Once there is life, there is hope' Ebola survivors' experiences, behaviours and attitudes in Sierra Leone, 2015. BMJ Glob Health. (2016) 1:e000108. doi: 10.1136/bmjgh-2016-000108

43. Azman AB, Sararaks S, Rugayah B, Low LL, Azian AA, Geeta S, et al. Quality of life of the Malaysian general population: results from a postal survey using the SF-36. Med J Malaysia. (2003) 58:694-711.

44. Fryback DG, Dunham NC, Palta M, Hanmer J, Buechner J, Cherepanov $\mathrm{D}$, et al. US norms for six generic health-related quality-of-life indexes from the national health measurement study. Med Care. (2007) 45:116270. doi: 10.1097/MLR.0b013e31814848f1

45. Roser K, Mader L, Baenziger J, Sommer G, Kuehni CE, Michel G. Health-related quality of life in Switzerland: normative data for the SF-36v2 questionnaire. Qual Life Res. (2019) 28:196377. doi: 10.1007/s11136-019-02161-5

46. Sabbah I, Drouby N, Sabbah S, Retel-Rude N, Mercier M. Quality of life in rural and urban populations in Lebanon using SF-36 health survey. Health Qual Life Outcomes. (2003) 1:30. doi: 10.1186/1477-7525-1-30

47. Aboabat A, Qannam H, Bjorner JB, Al-Tannir M. Psychometric validation of a Saudi Arabian version of the sf-36v2 health survey and norm data for Saudi Arabia. J Patient Rep Outcomes. (2020) 4:67. doi: 10.1186/s41687-020-00233-6

48. Jutzeler CR, Bourguignon L, Weis CV, Tong B, Wong C, Rieck B, et al. Comorbidities, clinical signs and symptoms, laboratory findings, imaging features, treatment strategies, and outcomes in adult and pediatric patients with COVID-19: a systematic review and meta-analysis. Travel Med Infect Dis. (2020) 37:101825. doi: 10.1016/j.tmaid.2020.101825

49. Ouanes S, Kumar R, Doleh ESI, Smida M, Al-Kaabi A, Al-Shahrani $\mathrm{AM}$, et al. Mental health, resilience, and religiosity in the elderly under COVID-19 quarantine in Qatar. Arch Gerontol Geriatr. (2021) 96:104457. doi: 10.1016/j.archger.2021.104457

50. Wang $\mathrm{Q}, \mathrm{Xu} \mathrm{R}$, Volkow ND. Increased risk of COVID-19 infection and mortality in people with mental disorders: analysis from electronic health records in the United States. World Psychiatry. (2021) 20:12430. doi: 10.1002/wps.20806

51. Johnson LC, Beaton R, Murphy S, Pike K. Sampling bias and other methodological threats to the validity of health survey research. Int J Stress Manag. (2000) 7:247-67. doi: 10.1023/A:1009589812697

Conflict of Interest: The authors declare that the research was conducted in the absence of any commercial or financial relationships that could be construed as a potential conflict of interest.

Publisher's Note: All claims expressed in this article are solely those of the authors and do not necessarily represent those of their affiliated organizations, or those of the publisher, the editors and the reviewers. Any product that may be evaluated in this article, or claim that may be made by its manufacturer, is not guaranteed or endorsed by the publisher.

Copyright (C) 2021 Ouanes, Al-Amin, Hussein, Khan, Al Shahrani, David, Wali, Thapur, Karim, Al Maslamani, Al-Ansari and Ghuloum. This is an open-access article distributed under the terms of the Creative Commons Attribution License (CC $B Y)$. The use, distribution or reproduction in other forums is permitted, provided the original author(s) and the copyright owner(s) are credited and that the original publication in this journal is cited, in accordance with accepted academic practice. No use, distribution or reproduction is permitted which does not comply with these terms. 